KCL-TH-00-48

hep-th/0008047

October 28, 2018

\title{
Chiral Superfields in IIB Supergravity
}

\author{
P. Heslop and P.S. Howe \\ Department of Mathematics \\ King's College, London
}

\begin{abstract}
The field strength superfield of IIB supergravity on $A d S_{5} \times S^{5}$ is expanded in harmonics on $S^{5}$ with coefficients which are $D=5, N=8$ chiral superfields. On the boundary of $A d S_{5}$ these superfields map to $D=4, N=4$ chiral superfields and both sets of superfields obey additional fourth-order constraints. The constraints on the $D=4, N=4$ chiral fields are solved using harmonic superspace in terms of prepotential superfields which couple in a natural way to composite operator multiplets of the boundary $N=4, D=4$ superconformal field theory.
\end{abstract}


A key feature of the AdS/CFT correspondence [1] is the simple fact that the symmetry group of $A d S$ spacetime is the same as the conformal group of the boundary Minkowski space, a feature which extends to supersymmetric theories in a natural way when they are formulated in superspace. Perhaps the clearest and best-established example of the correspondence is between IIB supergravity on $A d S_{5} \times S^{5}$ and $D=4, N=4$ supersymmetric $S U\left(N_{c}\right)$ Yang-Mills theory (in the large $N_{c}$ limit) on the boundary of $A d S_{5}$. The Kaluza-Klein spectrum of this supergravity theory was found some time ago [2], and in [3] a natural family of gauge-invariant composite operators in N=4 SYM was written down using a formulation of the theory in a

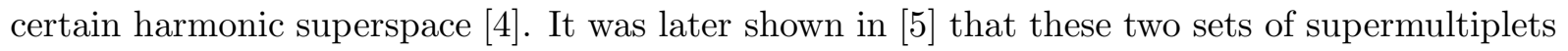
are in one-one correspondence. In this note we shall look at the relation between these two sets of supermultiplets in a very explicit fashion by tracing what happens to the linearised IIB field strength supermultiplet on $A d S_{5} \times S^{5}$ as one first expands it in terms of $S^{5}$ harmonics and then passes to the boundary to obtain a set of $D=4, N=4$ chiral superfields. We show that the constraints satisfied by this set of field strength superfields can easily be solved in harmonic superspace in terms of a family of Grassmann-analytic prepotential superfields (one for each chiral field) and the picture is completed by coupling the prepotentials to generalised current multiplets. The constraints on the latter, arising from the gauge invariances of the prepotentials, are precisely those that are obeyed by the family of $N=4$ SYM composite operators mentioned above.

The basic geometrical set-up is summarised by the following diagram:

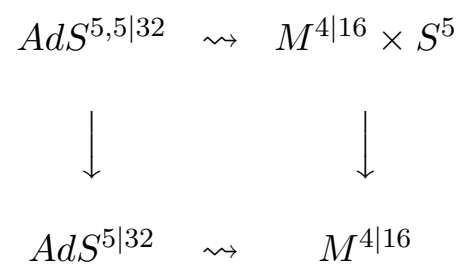

where the squiggly arrows denote passing to the boundary. Each of these superspaces is a coset space of the supergroup $P S U(2,2 \mid 4)$, with the notation indicating the (even|odd) dimensions. Thus $A d S^{5,5 \mid 32}$ denotes the superspace whose body is $A d S_{5} \times S^{5}$ and which has 32 odd dimensions while $A d S^{5 \mid 32}$ is the $D=5, N=8$ superspace which has $A d S_{5}$ for its body and which has 32 odd dimensions. The boundary space on the bottom row is $D=4, N=4$ Minkowski superspace. The isotropy group of $A d S^{5,5 \mid 32}$ is $\operatorname{Spin}(1,4) \times U S p(4)$ while that of $A d S^{5 \mid 32}$ is $\operatorname{Spin}(1,4) \times S U(4)$ and the former fibres over the latter with fibre $S^{5}$. The boundary superspaces are most easily described by viewing $P S U(2,2 \mid 4)$ as the $D=4, N=4$ superconformal group. The generators of the corresponding Lie superalgebra are $\{D, P, K, M, N \mid Q, S\}$ standing for dilations $(D)$, translations $(P)$, Lorentz transformations $(M)$, internal $S U(4)$ transformations $(N)$, and $Q$ and $S$-supersymmetry transformations, all in four dimensional spacetime. The istropy group of $M^{4 \mid 16}$ is generated by $\{D, K, M, N \mid S\}$ while the isotropy group of $M^{4 \mid 16} \times S^{5}$ is generated by $\left\{D, K, M, N^{\prime} \mid S\right\}$, where $N^{\prime}$ denotes the generators of the $U S p(4)$ subgroup of $S U(4)$.

We recall that IIB supergravity has the following bosonic component fields: the graviton, a complex scalar, a complex two-form potential, and a real four-form potential whose five-form 
field-strength is self-dual. The fermions are a complex Weyl gravitino and a complex Weyl spinor. In the linearised theory these fields can be put together in a chiral superfield $\mathbb{A}$ satisfying a fourth-order constraint which is schematically of the form $D^{4} \mathbb{A} \sim \bar{D}^{4} \overline{\mathbb{A}}[\mathbb{6}]$. In fact, the $D^{4}$ component of $\mathbb{A}$ can be decomposed into two fields which are irreducible tensors of $S O(1,9)$ with 770 and 1050 components. The former is the Weyl tensor and is real while the latter is purely imaginary. The five-form field strength only enters into the chiral superfield at $\theta^{4}$ level via its derivative and the resulting six-index tensor, antisymmetric and self-dual on five of the indices, but not antisymmetric on all six, transforms under the 1050 representation of $S O(1,9)$. In the full theory, there is still a complex scalar superfield but it takes its values in the coset space $U(1) \backslash S L(2, \mathbb{R})$. The geometrical field strength tensors, the torsion and curvature and three-form and five-form field strengths, constructed from the supervielbein, the connection and the superspace two- and four-form potentials, are described in terms of a spinor superfield $\Lambda$ whose leading component is the spinor field of the supergravity multiplet and a five-index antisymmetric tensor superfield $G_{a b c d e}$ whose leading component is the covariantised spacetime five-form field strength. These superfields are of course not independent since together they describe the supergravity multiplet.

The homogeneous superspace $A d S^{5,5 \mid 32}$ has been described in references [7, 8, 9, 10]. Since it is a coset one can use standard homogeneous space techniques to construct the components of the torsion and curvature tensors. In fact, it turns out that for this superspace the spinor superfield $\Lambda$ vanishes while the superfield $G_{a b c d e}$ has constant components which are non-vanishing only if all of the indices are in the range 0 to 4 or in the range 5 to 9 , the non-vanishing components being proportional to five-dimensional epsilon tensors. If one expands about this background, then to first order, the theory is still described by a chiral scalar superfield. Moreover, since the anticommutator of two fermionic covariant derivatives has the same form as in flat space (with the difference that the spacetime derivative must be amended), it follows that $D^{4} \mathbb{A}$ still falls into the same two representations of the Lorentz group as before, and it is not difficult to show that the same fourth-order constraint holds.

As we remarked above $A d S^{5,5 \mid 32}$ can be regard as a fibre bundle over $A d S^{5 \mid 32}$ with fibre $S^{5}$. This means that we can expand out the IIB chiral superfield $\mathbb{A}$ in harmonics on the five-sphere. The expansion has the form

$$
\mathbb{A}=\sum_{k=0}^{\infty} n^{R_{1}} \ldots n^{R_{k}} \mathcal{A}_{R_{1} \ldots R_{k}}^{(k)}
$$

Here $n^{R}, R=1, \ldots 6$, is a unit vector on the sphere and each of the tensors $\mathcal{A}^{(k)}$ is symmetric and traceless on its $S O(6)$ indices.

The component superfields $\mathcal{A}^{(k)}$ are all chiral superfields on $A d S^{5 \mid 32}$ and satisfy fourth-order reality conditions. However, for the components with indices the spinorial covariant derivative is altered by a term involving the $S O(6)$ connection of the fibred superspace. Clearly this term depends on the representation the component is in. The lowest component superfield $\mathcal{A}^{(0)}$ is the field strength superfield of linearised $N=8, D=5$ gauged supergravity.

\footnotetext{
${ }^{1}$ This fact was observed by N. Berkovits (private communication); it can also be derived from the full theory.
} 
We can pass to the boundary in the following way (see [8, 10] for related work). For $A d S^{5 \mid 32}$ we may choose the coset representative $s(r, \varphi, x, \theta)$ to be of the form

$$
s=e^{\varphi \cdot S} e^{\ln r D} g(x, \theta) .
$$

Here $g(x, \theta)$ is the standard coset representative for $D=4, N=4$ Minkowski superspace, $\varphi$ denotes the odd coordinates associated with $S$-supersymmetry, and $(r, x)$ are coordinates for $A d S_{5}$. In these coordinates the metric of $A d S_{5}$ itself is

$$
d s^{2}=r^{-2}\left(d r^{2}+d x^{2}\right)
$$

where $d x^{2}$ denotes the flat $D=4$ Minkowski space metric. We will take the boundary to be at $\varphi=0, r \rightarrow 0$.

$A d S^{5 \mid 32}$ has isotropy group $\operatorname{Spin}(1,4) \times S U(4)$, with Lie algebra generated by $\left\{\frac{1}{2}(K-P), M, N\right\}$ The remaining coset generators are given by $\left\{\frac{1}{2}(K+P), D \mid Q, S\right\}$. When $\varphi=0$

$$
d s s^{-1}=\frac{1}{r} e^{a} P_{a}+\frac{1}{\sqrt{r}}\left(e^{\alpha_{q}} Q_{\alpha_{q}}\right)+\left(d \varphi^{\alpha_{s}} S_{\alpha_{s}}\right)+\frac{d r}{r} D
$$

where $e^{A}=\left(e^{a}, e^{\alpha_{q}}\right)$ is the standard one form basis in flat $\mathcal{N}=4$ superspace, given by:

$$
d g g^{-1}=e^{a} P_{a}+\left(e^{\alpha_{q}} Q_{\alpha_{q}}\right) .
$$

From (5) we see that the one form basis of $A d S^{5 \mid 32}$ when $\varphi=0$ is

$$
E^{a}=\frac{1}{r} e^{a} \quad E^{4}=\frac{d r}{r} \quad E^{\alpha_{q}}=\frac{1}{\sqrt{r}} e^{\alpha_{q}} \quad E^{\alpha_{s}}=d \varphi^{\alpha_{s}}
$$

We wish to compare the bulk superisometries of $A d S^{5 \mid 32}$ with superconformal transformations of $\mathcal{N}=4$ Minkowski space on the boundary. Here we introduce the following indices: $M=\left(m, \mu_{q}\right)$, $M^{\prime}=\left(m, 4, \mu_{q}, \mu_{s}\right)$, and similarly for the flat indices $A, A^{\prime}$. Superconformal transformations act on the coordinates $z=(x, \theta)$ by $\delta_{s c} z^{M}=\xi^{M}$, where

$$
g \mathbf{T} g^{-1}=\xi^{a} P_{a}+\left(\xi^{\alpha_{q}} Q_{\alpha_{q}}\right)+\left(\omega^{\alpha_{s}} S_{\alpha_{s}}\right)+\lambda D+\ldots
$$

Here $\mathbf{T}$ is an arbitrary infinitesimal element of the Lie algebra, and $\xi^{M}=\xi^{A} e_{A}{ }^{M}$. The dots represent other elements of the isotropy Lie algebra. The isometries of $A d S^{5 \mid 32}$ act on the coordinates $Z^{\prime}=(x, r, \theta, \varphi)$ of $A d S^{5 \mid 32}$ by $\delta_{a d s} Z^{M^{\prime}}=\Xi^{M^{\prime}}=\Xi^{A^{\prime}} E_{A^{\prime}} M^{\prime}$ where

$$
s \mathbf{T} s^{-1}=\Xi^{A^{\prime}} \mathbf{T}_{A^{\prime}}+\Omega^{r} \mathbf{T}_{r} .
$$

On the right hand side we have split the Lie algebra up into a coset part and an isotropy group part for $A d S^{5 / 32}$. Using (3) and (8) we find that at $\varphi=0$

$$
s \mathbf{T} s^{-1}=\frac{1}{r} \xi^{a} P_{a}+\frac{1}{\sqrt{r}}\left(\xi^{\alpha_{q}} Q_{\alpha_{q}}\right)+\sqrt{r}\left(\omega^{\alpha_{s}} S_{\alpha_{s}}\right)+\lambda D+\ldots
$$


This gives us the following at $\varphi=0$

$$
\begin{aligned}
\delta_{a d s} x & =\delta_{s c} x+O\left(r^{2}\right) \\
\delta_{a d s} \theta & =\delta_{s c} \theta \\
\delta_{a d s} \varphi & =\sqrt{r} \omega \\
\delta_{a d s} r & =r \lambda .
\end{aligned}
$$

The $O\left(r^{2}\right)$ term appears from changing from the superconformal basis to the AdS basis of the Lie algebra. We see that the action of the isometries of the bulk space on the coordinates $(x, \theta)$ is equivalent to superconformal transformations on $N=4$ super Minkowski space. Also the action of the isometries transverse to this boundary disappears, and so setting $\varphi=0$ is a consistent thing to do. (Note that this is not the case if we instead choose the coset representative $s=e^{\ln r D} e^{\varphi \cdot S} g(x, \theta)$. In this case one has to argue that the dependence on $\varphi$ of the bulk fields tends to zero.)

A similar construction holds for $A d S^{5,5 \mid 32}$ except that we also have to include coordinates for $S^{5}$. In the boundary limit $r \rightarrow 0, \varphi=0$ the dependence of the chiral superfields on half of the odd coordinates disappears and we thus end up with a family of $D=4, N=4$ chiral superfields $\left\{A_{R_{1} \ldots R_{k}}^{(k)}\right\}, k=0,1,2, \ldots$. These superfields obey the chirality constraint

$$
\bar{D}_{\dot{\alpha}}^{i} A^{(k)}=0
$$

where we have adopted two-component spinor notation for four-dimensional spacetime and where the index $i$ runs from 1 to 4 . The fourth-order constraints become

$$
D_{\left\{R_{1} R_{2}\right.}^{4} A_{\left.R_{3} \ldots R_{k+2}\right\}}^{(k)}=\overline{D_{\left\{R_{1} R_{2}\right.}^{4} A_{\left.R_{3} \ldots R_{k+2}\right\}}^{(k)}}
$$

where the curly brackets denote traceless symmetrisation. The derivative $D_{R S}^{4}$ is formed from $\left(D_{\alpha i}\right)^{4}$ by taking the component which is a Lorentz scalar and which transforms under the real 20-dimensional representation of $S U(4)$; in $S O(6)$ notation this projection corresponds to a second-rank symmetric traceless tensor. The lowest superfield in this set, $A^{(0)}$, is the field strength superfield of $D=4, N=4$ conformal supergravity. Note that while the $N=8, D=5$ superfields $\mathcal{A}^{(k)}$ are on-shell fields the $N=4, D=4$ superfields $A^{(k)}$ are off-shell.

In order to complete the picture we now need to translate the fourth-order constraint (13) into harmonic superspace language [11]. The harmonic superspace we shall use is $M \times S(U(2) \times$ $U(2)) \backslash S U(4)$ where $M$ denotes $N=4$ super Minkowski space. We shall employ the standard GIKOS trick of working on $M \times S U(4)$ and demanding that the behaviour of the fields with respect to the isotropy group is fixed. We write $u \in S U(4)$ as $u_{I}{ }^{i}=\left(u_{r}{ }^{i}, u_{r^{\prime}}{ }^{i}\right)$ in index notation, and denote the inverse of $u$ by $u_{i}{ }^{I}=\left(u_{i}{ }^{r}, u_{i}{ }^{r^{\prime}}\right)$. The capital $I$ index is understood to be acted on by the isotropy group and thus splits naturally into $r=1,2$ and $r^{\prime}=3,4$. With the aid of $u$ and its inverse we can convert $S U(4) i$ indices into isotropy group $I$ indices and vice versa. Differentiation on the group itself is done using the right-invariant derivatives $D_{I}{ }^{J}$, with $D_{I}^{I}=0$. From the fact that these derivatives generate the left action of the group on itself we find 


$$
D_{I}^{J} u_{K}^{k}=\delta_{K}^{J} u_{I}^{k}-\frac{1}{4} \delta_{I}^{J} u_{K}^{k}
$$

The set of right-invariant derivatives splits into the isotropy group derivatives $\left\{D_{r}{ }^{s}, D_{r^{\prime}} s^{\prime}, D_{o}\right\}$ and coset derivatives $\left\{D_{r} s^{\prime}, D_{r^{\prime}}{ }^{s}\right\}$. The isotropy derivatives have been written in $\mathfrak{s u}(2) \oplus \mathfrak{s u}(2) \oplus$ $\mathfrak{u}(1)$ notation (so that $D_{r}^{r}=D_{r^{\prime}}{ }^{\prime}=0$ ), and our normalisation is such that

$$
D_{o} u_{r}^{i}=\frac{1}{2} u_{r}^{i} \quad D_{o} u_{r^{\prime}}{ }^{i}=-\frac{1}{2} u_{r^{\prime}}{ }^{i}
$$

The coset space $S(U(2) \times U(2)) \backslash S U(4)$ is a compact complex manifold with complex dimension four (it is the Grassmannian of two-planes in $\mathbb{C}^{4}$ ), and the derivatives $D_{r}{ }^{s^{\prime}}$ can be thought of as the components of the $\bar{\partial}$ operator on this space while the derivatives $D_{r^{\prime}}$ s are the complex conjugates. The introduction of harmonics allows the possibility of having superfields which satisfy generalised chirality constraints; in the present case, the derivatives $D_{\alpha r}=u_{r}{ }^{i} D_{\alpha i}$ and $\bar{D}_{\dot{\alpha}}^{r^{\prime}}=\bar{D}_{\dot{\alpha}}{ }^{i} u_{i}{ }^{r^{\prime}}$ anticommute with each other and commute with $D_{r}{ }^{s^{\prime}}$. A field $F$ on harmonic superspace which satisfies

$$
D_{\alpha r} F=\bar{D}_{\dot{\alpha}}^{r^{\prime}} F=0
$$

is called Grassmann analytic, or G-analytic, while a field which satisfies $D_{r}{ }^{\prime} F=0$ is called harmonic analytic, or $\mathrm{H}$-analytic. A field which is both $\mathrm{G}$ - and $\mathrm{H}$-analytic will be called an analytic superfield.

For the following discussion it will be useful to define the object $u^{i j}=-u^{j i}$ by

$$
u^{i j}:=\frac{1}{2} \epsilon^{r s} u_{r}^{i} u_{s}^{j}
$$

The fact that $u \in S U(4)$ then implies that

$$
\begin{aligned}
u_{i j} & :=\frac{1}{2} \epsilon_{i j k l} u^{k l} \\
& =\frac{1}{2} \epsilon_{r^{\prime} s^{\prime}} u_{i}^{{ }^{\prime}{ }^{\prime}} u_{j}^{s^{\prime}}
\end{aligned}
$$

We shall also employ the following abbreviations for fourth-order Grassmann derivatives which are Lorentz scalars,

$$
\begin{array}{rll}
D^{4}:=\left(D_{\alpha r}\right)^{4} & D^{\prime 4}:=\left(D_{\alpha r^{\prime}}\right)^{4} \\
\bar{D}^{4}:=\left(\bar{D}_{\dot{\alpha}}{ }^{r}\right)^{4} & \bar{D}^{\prime 4}:=\left(\bar{D}_{\dot{\alpha}}^{r^{\prime}}\right)^{4}
\end{array}
$$

The final technicality we shall need is the notion of harmonic conjugation [1] which combines a generalisation of the antipodal map for $\mathbb{C}^{1}$ to the coset $S(U(2) \times U(2)) \backslash S U(4)$ with complex conjugation. We shall denote this operation by a tilde. For the $u$ 's we have 


$$
\begin{aligned}
& \left(\tilde{u_{r}}{ }^{i}\right)=u_{i}{ }^{r^{\prime}} \\
& \left(\tilde{u_{r^{\prime}}}{ }^{i}\right)=-u_{i}^{r}
\end{aligned}
$$

while for any $u$-independent object we use ordinary complex conjugation. It follows that Ganalyticity and $\mathrm{H}$-analyticity are both preserved by harmonic conjugation and that

$$
\left(\tilde{u^{i j}}\right)=u_{i j}
$$

Returning to the boundary chiral field strength superfields, we can define the following family of objects

$$
\begin{aligned}
C^{(k)}: & =u^{i_{1} j_{1}} \ldots u^{i_{k+2} j_{k+2}} D_{i_{1} j_{1}, i_{2} j_{2}}^{4} A_{i_{3} j_{3}, \ldots i_{k+2} j_{k+2}}^{(k)} \\
& \sim u^{i_{1} j_{1}} \ldots u^{i_{k} j_{k}} D^{4} A_{i_{1} j_{1}, \ldots i_{k} j_{k}}^{(k)}
\end{aligned}
$$

where we have converted the $S O(6)$ indices $R$ into antisymmetric self-dual pairs of $S U(4)$ indices and then contracted all these pairs with $u^{i j}$ 's. We claim that each $C^{(k)}$ is analytic. H-analyticity is immediate, since $u^{i j}$ is annihilated by $D_{r}{ }^{\prime}$. G-analyticity follows because the presence of the $u$ 's implies that the projection of $D^{4}$ involves only the derivatives $D_{\alpha r}$. Since the product of five such derivatives vanishes identically, we see that $D_{\alpha r} C^{(k)}=0$, while $\bar{D}_{\dot{\alpha}}^{r^{\prime}}$ can be anti-commuted past the $D$ 's to act on the chiral field $A$ whereupon it gives zero. The fourth-order constraint on the field $A^{(k)}$ (with $k S O(6)$ indices) can therefore be written in the form

$$
C^{(k)}=\tilde{C}^{(k)}
$$

Since this constraint is non-dynamical it can be imposed by a Lagrange multiplier superfield, $V^{(k)}$, say, which may be taken to be G-analytic. The free action for $A^{(k)}$ can therefore be writtenf

$$
S^{(k)}=\left(\int d^{4} x d^{8} \theta \frac{1}{2}\left(A^{(k)}\right)^{2}\right)+c . c .+\int d \mu V^{(k)}\left(C^{(k)}-\tilde{C}^{(k)}\right)
$$

In this formula, the first integral is an $N=4$ chiral integral while the second one is a harmonic superspace integral. The harmonic superspace measure $d \mu$ is defined by

$$
d \mu:=d^{4} x d u D^{\prime 4} \bar{D}^{4}
$$

where $d u$ denotes the standard measure on the coset space. If we vary the above action with respect to $A^{(k)}$, which is here regarded as an otherwise unconstrained chiral superfield, we get an expression for $A^{(k)}$ in terms of the prepotential $V^{(k)}$. It is

\footnotetext{
${ }^{2}$ Similar methods can be used to find prepotentials in Minkowski superspace, as in 12
} 


$$
A_{i_{1} j_{1}, \ldots i_{k} j_{k}}^{(k)}=\int d u u_{i_{1} j_{1}} \ldots u_{i_{k} j_{k}} \bar{D}^{4} V^{(k)}
$$

This formula generalises in a natural way the expression for the chiral $N=2$ Maxwell field strength multiplet in terms of a G-analytic charge two potential [13].

We note that $u^{i j}$ has $U(1)$ charge 1 so that $C^{(k)}$ has $U(1)$ charge $k+2$. Since the charges of $D^{4}, D^{\prime 4}, \bar{D}^{4}, \bar{D}^{\prime 4}$ are $2,-2,-2$ and 2 respectively the charge of the harmonic measure is -4 , so that $V^{(k)}$ has charge $-k+2$. We note further that, since $C^{(k)}$ is both G- and H-analytic, the action $S^{(k)}$ has an abelian gauge invariance of the form

$$
V^{(k)} \rightarrow V^{(k)}+D_{r}^{s^{\prime}} X_{s^{\prime}}^{(k) r}
$$

where the parameter superfield $X^{(k)}$ is G-analytic.

The prepotentials $V^{(k)}$ couple naturally to generalised currents $\mathcal{O}_{k+2}$ via the Noether coupling

$$
S_{\text {Noeth }}^{(k)}=\int d \mu V^{(k)} \mathcal{O}_{k+2}
$$

Clearly the current $\mathcal{O}_{k+2}$ has the same properties as the corresponding constraint $C^{(k)}$, in particular $\mathcal{O}_{k}$ has $U(1)$ charge $k$.

The currents can be realised explicitly as composite operators in $N=4 S U\left(N_{c}\right)$ Yang-Mills theory. We recall that the $N=4$ Maxwell field strength supermultiplet is described in $N=4$ super Minkowski space by the Sohnius superfield $W_{i j}$ [14] which is antisymmetric and self-dual and which satisfies

$$
\begin{aligned}
D_{\alpha i} W_{j k} & =D_{[\alpha i} W_{j k]} \\
\bar{D}_{\dot{\alpha}}^{i} W_{j k} & =-\frac{2}{3} \delta_{[j}^{i} \bar{D}_{\dot{\alpha}}^{l} W_{k] l}
\end{aligned}
$$

These constraints can be translated into harmonic superspace by introducing the field $W:=$ $u^{i j} W_{i j}$ [4]. This is an analytic superfield which has $U(1)$ charge $1\left(D_{o} W=W\right)$ and which is real with respect to harmonic conjugation, $W=\tilde{W}$. Conversely one can show that that such an analytic superfield is equivalent to the Sohnius superfield. In the non-Abelian theory the field strength superfield $W$ is $\mathrm{H}$-analytic, but the $\mathrm{G}$-analyticity condition has to be made covariant with respect to the gauge group. However, the gauge-covariant operators $\mathcal{O}_{k}:=\operatorname{tr}\left(W^{k}\right)$ are analytic in the usual sense, and so provide the desired family of generalised currents which couple to the prepotentials $V^{(k)}$.

\section{Acknowledgement}

This research was supported in part by PPARC SPG grant 613. 


\section{References}

[1] J. Maldacena, The large $N$ limit of superconformal field theories and supergravity, Adv. Theor. Math. Phys. 2 (1998) 231-252, hep-th/9711200; S.S. Gubser, I.R. Klebanov and A.M. Polyakov, Gauge theory correlators from noncritical String theory, Phys. Lett. B428 (1998) 105, hep-th/9802109; E. Witten, Anti-de Sitter space and holography, Adv. Theor. Math. Phys. 2 (1998) 253-291, hep-th/9802150.

[2] M. Gunaydin and N. Marcus, Class. Quant. Grav. 2 (1985) L11; M. Gunaydin, L.J. Romans and N. Warner, Phys. Lett. 154B (1985) 268.

[3] P.S. Howe and P.C. West, Nonperturbative Green's functions in theories with extended superconformal symmetry Int. J. Mod. Phys. A14 (1999) 2659-2674, hep-th/9509140.

[4] G.G. Hartwell and P.S. Howe $(N, p, q)$ harmonic superspace, Int J. Mod. Phys 10, (1995) 3901-3919.

[5] L Andrianopoli and S Ferrara $K-K$ excitations on $A d S_{5} \times S^{5}$ and $N=4$ primary superfields, Phys. Lett. B430 (1998) 248-253.

[6] P.S. Howe and P.C. West, The complete $N=2, d=10$ supergravity, Nucl. Phys. B238 (1984) 181-220.

[7] R.R. Metsaev and A. Tseytlin, Type IIB Superstring Action in $A d S_{5} \times S^{5}$, Nucl. Phys B533 (1998) 109, hep-th/9805028.

[8] R. Kallosh, J. Rahmfeld and A. Rajaraman, Near Horizon Superspace, JHEP 9809 (1998) 002, hep-th/98050217

[9] P. Claus, J. Tannenhauser, H. Robins, J. Rahmfeld and Y. Zunger, Isometries in anti-de Sitter and Conformal Superspaces, hep-th/0007099.

[10] H. Oguri, J. Rahmfeld, H. Robins and J. Tannenhauser, Holography in Superspace, hepth/0007104.

[11] A Galperin A, E Ivanov, S Kalitzin, V Ogievetsky and E Sokatchev, Unconstrained $N=2$ matter, Yang-Mills and supergravity theories in harmonic superspace, Class. Quantum Grav. 1 (1984) 469.

[12] P.S. Howe, K.S. Stelle and P.K. Townsend, Nucl. Phys. B181 (1981) 445; Nucl. Phys. B182 (1981) 332 .

[13] A. Galperin, E. Ivanov, V. Ogievetsky and E. Sokatchev, Harmonic superspace: key to $N=2$ supersymmetry theories, JETP Lett. 40 (1984) 912-916; B. Zupnik, The action of the supersymmetric $N=2$ gauge theory in harmonic superspace, Phys.Lett. B183 (1987) 175-176.

[14] M.F. Sohnius, Nucl. Phys. 136 (1978) 461. 